\title{
Does stereoscopic imaging improve the memorization of medical imaging by neurosurgeons? Experience of a single institution
}

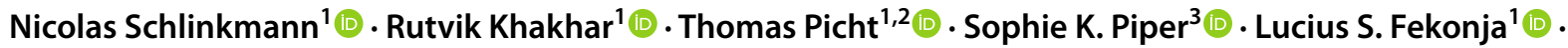 \\ Peter Vajkoczy ${ }^{1}$. Gueliz Acker ${ }^{1,4}$ (B)
}

Received: 18 May 2021 / Revised: 24 July 2021 / Accepted: 7 August 2021 / Published online: 22 September 2021

(C) The Author(s) 2021

\begin{abstract}
Stereoscopic imaging has increasingly been used in anatomical teaching and neurosurgery. The aim of our study was to analyze the potential utility of stereoscopic imaging as a tool for memorizing neurosurgical patient cases compared to conventional monoscopic visualization. A total of 16 residents and 6 consultants from the Department of Neurosurgery at Charité - Universitätsmedizin Berlin were recruited for the study. They were divided into two equally experienced groups. A comparative analysis of both imaging modalities was conducted in which four different cases were assessed by the participants. Following the image assessment, two questionnaires, one analyzing the subjective judgment using the 5-point Likert Scale and the other assessing the memorization and anatomical accuracy, were completed by all participants. Both groups had the same median year of experience (5) and stereoacuity ( $\leq 75 \mathrm{~s}$ of arc). The analysis of the first questionnaire demonstrated significant subjective superiority of the monoscopic imaging in evaluation of the pathology (median: monoscopic: 4; stereoscopic: $3 ; p=0.020$ ) and in handling of the system (median: monoscopic: 5 ; stereoscopic: $2 ; p<0.001$ ). The second questionnaire showed that the anatomical characterization of the pathologies was comparable between both visualization methods. Most participants rated the stereoscopic visualization as worse compared to the monoscopic visualization, probably due to a lack of familiarity with the newer technique. Stereoscopic imaging, however, was not objectively inferior to traditional monoscopic imaging for anatomical comprehension. Further methodological developments and incorporation in routine clinical workflows will most likely enhance the usability and acceptance of stereoscopic visualization.
\end{abstract}

Keywords $3 \mathrm{D} \cdot$ Stereoscopic $\cdot$ Memorization $\cdot$ Imaging $\cdot$ Neurosurgery

\section{Introduction}

Gueliz Acker

gueliz.acker@charite.de

1 Charité - Universitätsmedizin Berlin, corporate member of Freie Universität Berlin and Humboldt-Universität zu Berlin, Department of Neurosurgery, Charitéplatz 1, 10117 Berlin, Germany

2 Charité - Universitätsmedizin Berlin, corporate member of Freie Universität Berlin and Humboldt- Universität zu Berlin, Cluster of Excellence: "Matters of Activity. Image Space Material", Charitéplatz 1, 10117 Berlin, Germany

3 Charité - Universitätsmedizin Berlin, corporate member of Freie Universität Berlin and Humboldt-Universität zu Berlin, Institute of Biometry and Clinical Epidemiology, Charitéplatz 1, 10117 Berlin, Germany

4 Berlin Institute of Health at Charité - Universitätsmedizin Berlin, BIH Academy, Clinician Scientist Program, Charitéplatz 1, 10117 Berlin, Germany
In medicine, stereoscopic (stereo) screens have been increasingly utilized in teaching, preoperative planning, and also during surgery [1-5]. Due to depth perception created by the projection of a slightly different image to each eye, stereoscopic displays allow a more accurate visualization compared to three-dimensional (3D) images on two-dimensional (2D) displays [6]. In this regard, Harake et al. demonstrated that interactive stereoscopic visualization of three-dimensional echocardiography was preferred over conventional display by cardiologists, advanced cardiac trainees, and surgeons in viewing both simple and complex congenital cardiac lesions [7]. Surgical disciplines, like neurosurgery, orthopedics, or visceral surgery, are particularly interested in new ways to visualize complex anatomical relations during surgical planning $[1,4,5]$. However, the benefits of stereoscopic imaging for clinical routine are still controversial. 
While in a recent study we were able to show an advantage of stereoscopic imaging in the detection of challenging aneurysms, Stewart et al. could not detect any benefits with stereoscopic viewing of volume-rendered three-dimensional computed tomography angiograms (CT-A) in the characterization of cerebral aneurysms compared with monoscopic (mono) viewing $[8,9]$.

Memorization of the individual anatomy is particularly important for surgeons. For medical students, it has already been shown that hand-made drawings can be helpful in memorizing anatomical structures [10]. Furthermore, threedimensional programs have already proven to be advantageous in preoperative planning and in anatomical teaching $[11,12]$. However, Park et al. demonstrated that a threedimensional anatomical atlas for first-year medical students could not enhance the memorization of anatomical structures [13]. Importantly, a recent meta-analysis by Bogomolova et al. has shown that for learning anatomy, stereoscopic 3D is superior to monoscopic 3D [14]. The additional advantage of stereoscopic visualization compared to viewing 3D images on 2D displays, which is more prevalent in routine clinical practice, was highlighted in this report [14]. In terms of adoption, a high level of acceptance for stereoscopic 3D videos by students has been validated with an added benefit in anatomical understanding in a very recent study [15].

With regard to preoperative planning, the effectiveness of $3 \mathrm{D}$ visualization for neurosurgical interventions was reported back in 1996 [16]. Preoperative planning with interactive $3 \mathrm{D}$ computed tomography (CT) reconstruction has also been proven to be a useful method to enhance the surgeon's knowledge of the patient's individual anatomy in thoracoscopic lung surgery [17]. Similarly, a neurosurgeon's efficiency could be greatly improved by better understanding and memorizing the neuroanatomy prior to surgery using stereoscopic imaging. The aim of the present study is, therefore, to investigate, first on one hand, the subjective advantage of stereoscopic imaging for preoperative planning and, on the other hand, to evaluate the effect of stereoscopy on the memorization of anatomical information in patient cases of neurosurgery.

\section{Methods}

\section{Study design}

The study was performed in accordance with the ethical standards of the Declaration of Helsinki. The ethics committee of Charité - Universitätsmedizin Berlin approved the present retrospective analysis of data that was collected during department teaching (approval number, EA1/090/20). The imaging studies from patients treated at our department for subarachnoid hemorrhage from 2009 to 2018 and for brain tumor with preoperative fiber tracking from 2018 were reviewed to choose suitable cases for the teaching sessions. Two representative tumor and vascular surgical cases were selected based on predefined criteria, namely a tumor with involvement of the motor fiber tract and one with an affected language fiber tract, as well as an aneurysm in the anterior circulation and one in the posterior circulation.

Neurosurgeons in our department who attended this teaching session were included in the study. Participants were assigned to two balanced groups based on their experience level and stereopsis was tested with the Frisby Pocket Stereotest ${ }^{\mathrm{TM}}$. Group A first assessed a frontal brain tumor and a middle cerebral artery bifurcation aneurysm, while group B first assessed a central brain tumor and a vertebral artery aneurysm using the monoscopic visualization system. Shortly thereafter, the groups switched and assessed the cases of the other group using the stereoscopic visualization system (Fig. 1). All participants were briefly introduced to the handling of the stereoscopic visualization system before analyzing the cases with it. Each participant was given a maximum of 5 min per case to evaluate the images in each block for themselves. The time taken to identify the aneurysm during the image analysis was recorded. It is important to note that those participants who failed to detect an aneurysm were not included in the assessment of detection time. However, regarding the time to analyze the pathologies, all the participants for both visualization systems were included. In cases where the aneurysm could not be detected by the participant, the duration of the searching process (max. 5 min) was taken instead.

After analyzing the images with both visualization systems, all participants completed a subjective feedback questionnaire. The questionnaire obtained parameters such as the ability to recognize pathological and anatomical structures, amount of radiological information perceived, preferred visualization system, and confidence in using the visualization systems (supplemental Fig. 1). Participants were not informed about the content of the following day's re-evaluation but were notified that questions relating to the first day's procedure would be asked.

The following day, participants were asked to complete a questionnaire on the specifications of the pathology, such as the exact location, morphology, and invasion of associated tracts to assess the objective gain and retention of the information.

(supplemental Fig. 2-3).

\section{Characteristics of the selected surgical cases}

The tumor pathologies were two left-sided recurrent glioblastoma, one central with an invasion of the corticospinal tract (tumor 1; size: $13 \times 25 \times 20 \mathrm{~mm}$ ) and one in the middle frontal gyrus that extended to the frontal operculum involving the uncinate fascicle (tumor 2 ; size: $33 \times 18 \times 31 \mathrm{~mm}$ ) 


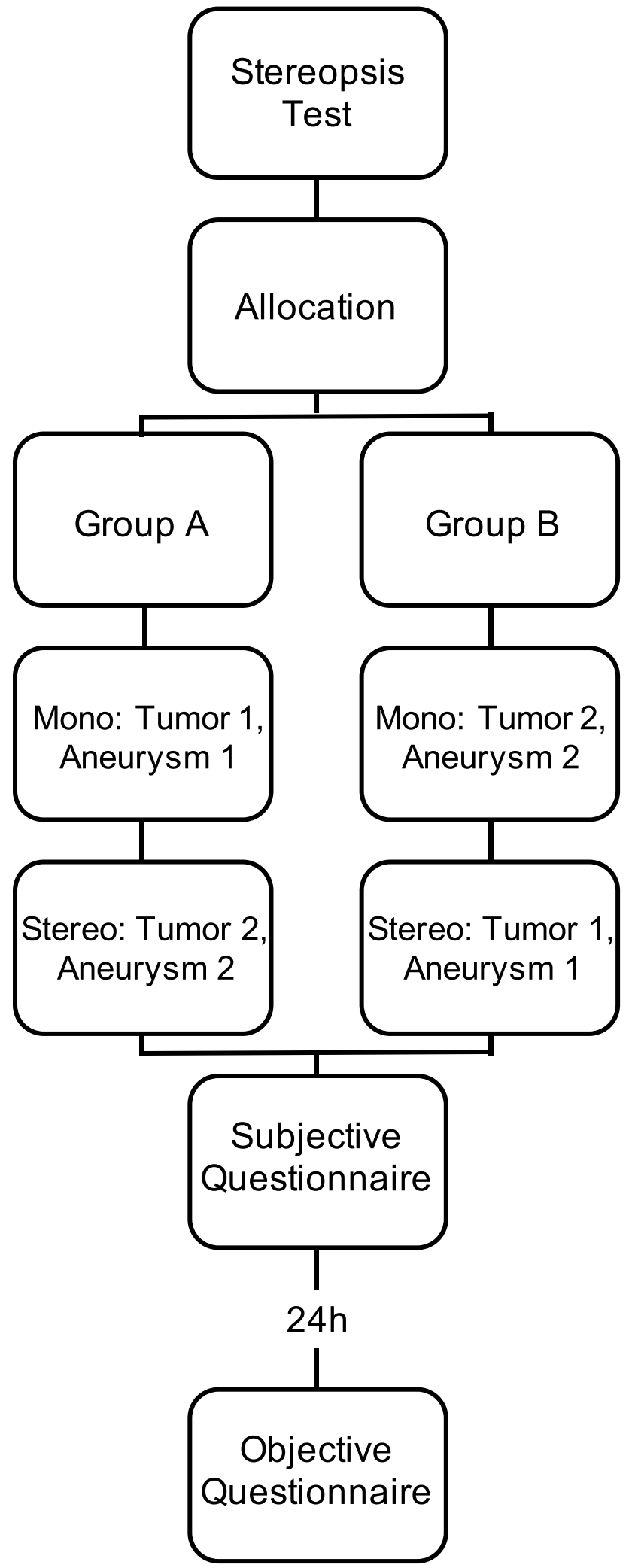

Fig. 1 Study design
(Fig. 2a-b). A right-sided vertebral artery fusiform aneurysm (aneurysm 1; $5 \times 7 \mathrm{~mm}$ ) and a right-sided middle cerebral artery saccular aneurysm (aneurysm 2; $9 \times 7 \mathrm{~mm}$ ) were selected for the neurovascular cases (Fig. 2c-d).

\section{Image processing and viewing}

Using the program VPI Reveal, version 1.5 (Vesalius Perfectus International BV, Eindhoven, the Netherlands), 3D volume rendering and display of the magnetic resonance imaging (MRI) and CT-A Digital Imaging and Communications in Medicine (DICOM) images were performed. VPI Reveal is a proprietary software based on inhouse developed 3D volume rendering technology using Raytracing technology and dedicated image post processing to facilitate optimal stereoscopic 3D display (Holographic 3D). The DICOM images were imported from the hospital's picture archiving and communication system (PACS) into the VP Reveal software, using the integrated import system, and the software automatically generated a 3D model. Subsequently, we could modify the visualization settings to improve the quality. For this purpose, each Hounsfield unit could be assigned with a specific gray value or a color. For the tumor and vascular system, an individual grayscale was chosen, while the imported fiber tracts were colored red. In each case, contrast and transparency of the images were adjusted so that an individual 3D model could be generated. This process required approximately $3 \mathrm{~min}$ per case. For image navigation, a 3D mouse (3Dconnexion SpaceMouse Compact) was used. The study was conducted at two different hospital sites, using a high-resolution liquid crystal display (LCD) monitor (Hyundai S465D; 3D LCD monitor, 46 in.; resolution: $1920 \times 1080 ; 60 \mathrm{~Hz})$ and a high-resolution lightemitting diode (LED) monitor (LG 55LA6208; 3D LED monitor, 55 in.; resolution: $1920 \times 1080 ; 200 \mathrm{~Hz} \mathrm{MCI)} \mathrm{for}$ 3D display, respectively. Commercially available polarized 3D glasses by Hyundai and LG were used. The VP Reveal was running on a Dell inc. Precision 7720 computer with an Intel Core ${ }^{\mathrm{TM}} \mathrm{i} 7-7920 \mathrm{HQ}(3.10 \mathrm{GHz})$ central processing unit and an NVIDIA Quadro P4000 graphics card. To evaluate the monoscopic (multiplanar reformation processed) MRI and CT-A images, a FUJITSU ESPRIMO D956 computer with an Intel Core ${ }^{\mathrm{TM}}$ i5-6500 $(3.20 \mathrm{GHz})$ central processing unit and an Intel HD Graphics 530 graphics card were used at both sites. The DICOM images were viewed via iPlan Net 3.0 (Brainlab, Munich) using a computer display monitor (Fujitsu B22T-7, 21.5 in.; resolution: $1920 \times 1080$ ).

\section{Statistical analysis}

Descriptive statistics were given as median with limits of the interquartile range (IQR) [25th-75th percentile]. Wilcoxon's signed-rank test was used to compare each 
Fig. 2 In the top row $(\mathbf{a}+\mathbf{b})$, an axial view of the T1-weighted magnetic resonance imaging of the central tumor (tumor 1), monoscopically with Brainlab (a) and stereoscopically with VP Reveal (b). The tract is shown in orange for Brainlab and red for VP Reveal. In the lower row $(\mathbf{c}+\mathbf{d})$, a coronal view of the computed tomography angiography of a middle cerebral artery bifurcation aneurysm (aneurysm 2), monoscopically with Brainlab (c) and stereoscopically with VP Reveal (d)
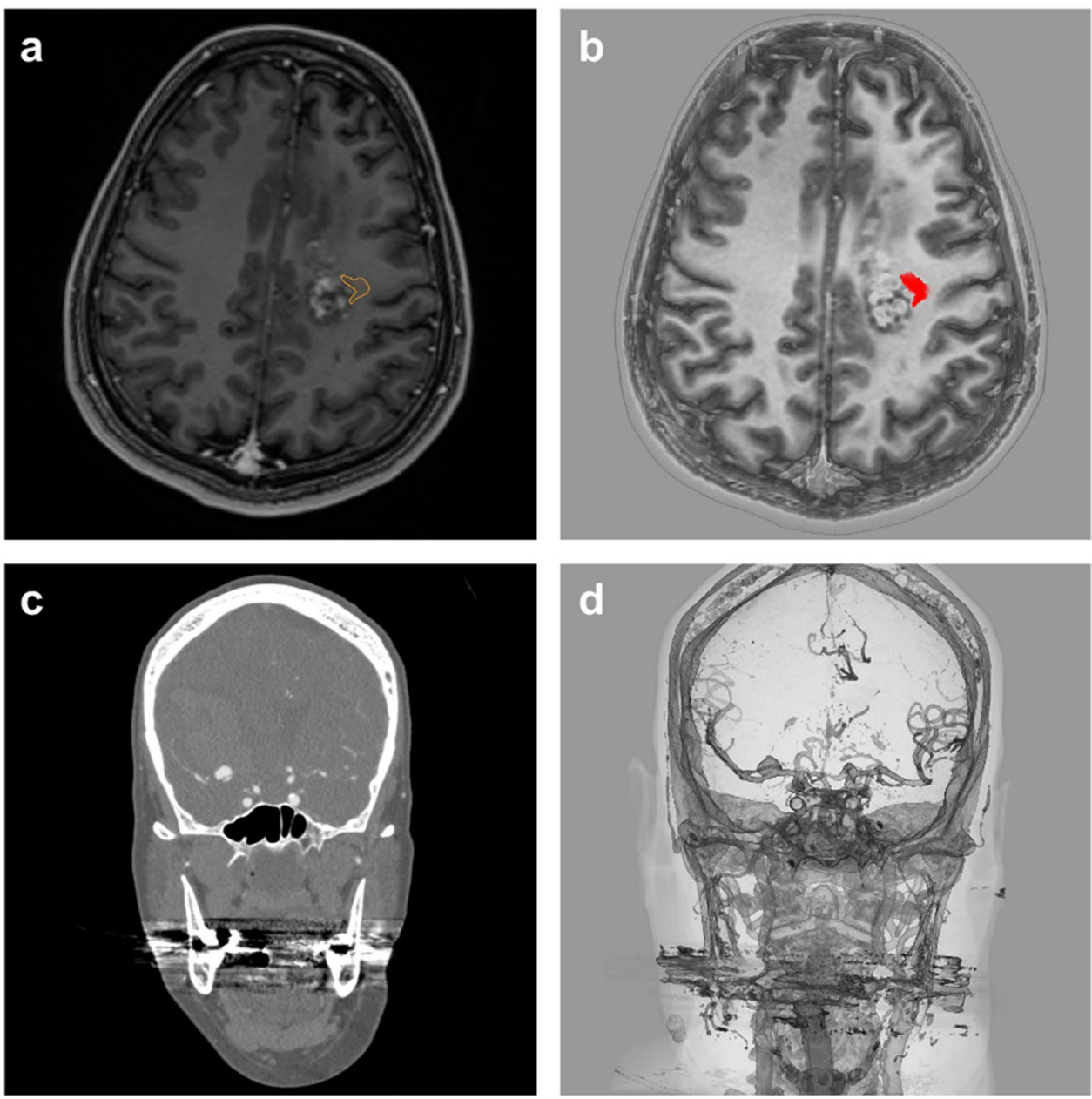

participant's subjective perception of monoscopic viewing with the corresponding stereoscopic viewing assessment, independent of group A or B. Objective perception was assessed as the number of correct responses per case for each participant in the second questionnaire. The total point of correct answers was 8 for each tumor and 7 for each aneurysm resulting from 8 and 7 different questions, respectively. The Mann-Whitney $U$ test was used to compare the objective perception rates between viewing modalities (monoscopic vs. stereoscopic) for each surgical case (tumor 1 , tumor 2 , aneurysm 1 , aneurysm 2). The years of experience were correlated with the objective perception for each viewing modalities (monoscopic vs. stereoscopic) using the Spearman rank correlation coefficient. All tests were 2 -sided, and $p<0.05$ was considered to indicate statistical significance. All tests should be understood as constituting an exploratory analysis and no adjustment for multiple testing was made. The statistical analyses were performed using SPSS, version 25 (International Business Machines Corporation (IBM), Armonk, NY). Graphs were made using GraphPad Prism, version 8 (GraphPad Software, San Diego, CA) and edited using PowerPoint, version 16
(Microsoft, Redmond, WA). Data and tables were managed with Excel, version 16 (Microsoft, Redmond, WA).

\section{Results}

\section{Characterization of the participants}

A total of 22 neurosurgeons participated as raters with different experience level (range: 1-12 years of experience), half of them $(n=11)$ were allocated into group $\mathrm{A}$ and the other half into group B. In each group, the median year of experience was 5 (Fig. 3). There were 10 female $(45.5 \%)$ and $12(54.5 \%)$ male participants. All participants had stereopsis with the same median of $75 \mathrm{~s}$ of arc (s arc) (Group A: median $75 \mathrm{~s}$ arc, IQR 75-90 s arc. Group B: median $75 \mathrm{~s}$ arc, IQR 75-75 s arc).

\section{Subjective analysis}

The questionnaire at the end of the first day, that documented the participants' subjective perception, revealed a significant 
Fig. 3 Comparison of both groups for years of experience with 11 participants in each group. The dots represent each participant's year of experience; the lines represent the median and the interquartile range (25th-75th percentile). Group A: years of experience: median 5, IQR 2-7. Group B: years of experience: median 5, IQR 3-8
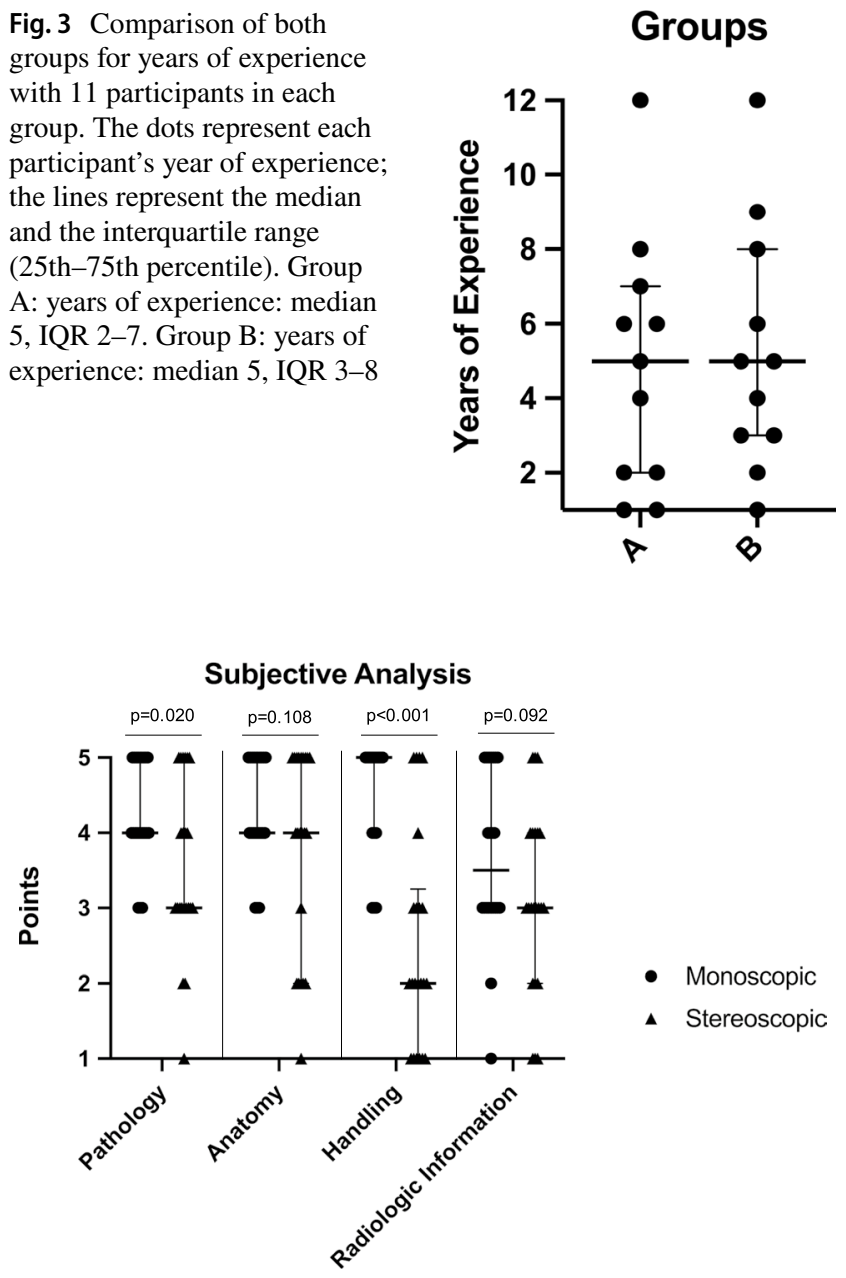

Fig. 4 Comparison of the subjective analysis of both image viewing modalities with 22 participants (Wilcoxon signed-rank test). The dots and triangles represent the points awarded by each participant for the various categories. Additionally, the lines represent the median and the interquartile range (25th-75th percentile). Pathology: mono: median 4, IQR 4-5; stereo: median 3, IQR 3-5. Anatomy: mono: median 4, IQR 4-5; stereo: median 4, IQR 2-5. Handling: mono: median 5, IQR 4-5; stereo: median 2, IQR 1-3.25. Radiologic information: mono: median 3.5, IQR 3-5; stereo: median 3, IQR 2-4 advantage of the monoscopic imaging in pathology assessment and subjective device handling (Fig. 4). The identification of the anatomy and the processing of the radiologic information did not differ between both modalities (Fig. 4). Overall, more than half of the participants $(n=14,63.6 \%)$ preferred the monoscopic imaging over stereoscopic, six (27.3\%) favored stereoscopy, while two (9.1\%) had no preference.

When comparing residents and consultants, the two modalities were rated similarly (Table 1). All but one of the medians differed no more than 0.5 points between both groups. The most relevant difference between residents and consultants was in the extraction of the radiologic information (median [IQR] residents: 3 [3-4.25], consultants: 4.5 [3.25-5]; $p=0.261$; Mann-Whitney $U$ test).

\section{Objective analysis}

In the second questionnaire on the following day, the objective gain of information was analyzed based on the points obtained by describing the localization and morphology of the pathologies. The evaluation of the pathologies did not differ significantly in any of the cases (Fig. 5). During the assessment, seven (31.8\%) participants could not detect the aneurysm in posterior circulation, three of group A (27.3\%, monoscopic) and four of group B (36.4\%, stereoscopic).

The comparison between residents and consultants highlighted that the various pathologies could be assessed equally well by both experience groups using both modalities without any significant differences in median (Table 2). The largest discrepancy between residents and consultants was in the objective analysis of tumor 2 by monoscopic visualization (median [IQR]: residents: 5.5 [4.75-6], consultants: 7 [6.5-7]; Mann-Whitney $U$ test $p=0.085$ ).

\section{Duration of the pathology assessment}

The time to analyze the pathologies was significantly longer using the stereoscopic imaging system for tumor 1, tumor 2, and aneurysm 2 (Fig. 6a), while it was comparable for the aneurysm 1 in the posterior circulation (Fig. 6a). For both
Table 1 Itemized subjective analysis comparison of residents and consultants

\begin{tabular}{|c|c|c|c|c|c|c|c|c|c|}
\hline & & \multicolumn{2}{|c|}{ Pathology } & \multicolumn{2}{|c|}{ Anatomy } & \multicolumn{2}{|c|}{ Handling } & \multicolumn{2}{|c|}{$\begin{array}{l}\text { Radiologic } \\
\text { information }\end{array}$} \\
\hline & & Mono & Stereo & Mono & Stereo & Mono & Stereo & Mono & Stereo \\
\hline \multirow[t]{3}{*}{ Residents $(n=16)$} & Median & 4 & 3 & 4.5 & 4 & 4.5 & 2 & 3 & 3 \\
\hline & 25th percentile & 4 & 3 & 4 & 3.5 & 3.75 & 1 & 3 & 2.75 \\
\hline & 75th percentile & 5 & 5 & 5 & 5 & 5 & 3.25 & 4.25 & 4 \\
\hline \multirow[t]{3}{*}{ Consultants $(n=6)$} & Median & 4 & 3.5 & 4 & 3.5 & 5 & 2.5 & 4.5 & 3.5 \\
\hline & 25 th percentile & 4 & 3 & 4 & 2.25 & 5 & 2 & 3.25 & 2.25 \\
\hline & 75th percentile & 4.75 & 4 & 4.75 & 4.75 & 5 & 3 & 5 & 4.75 \\
\hline
\end{tabular}




\section{Objective Analysis}
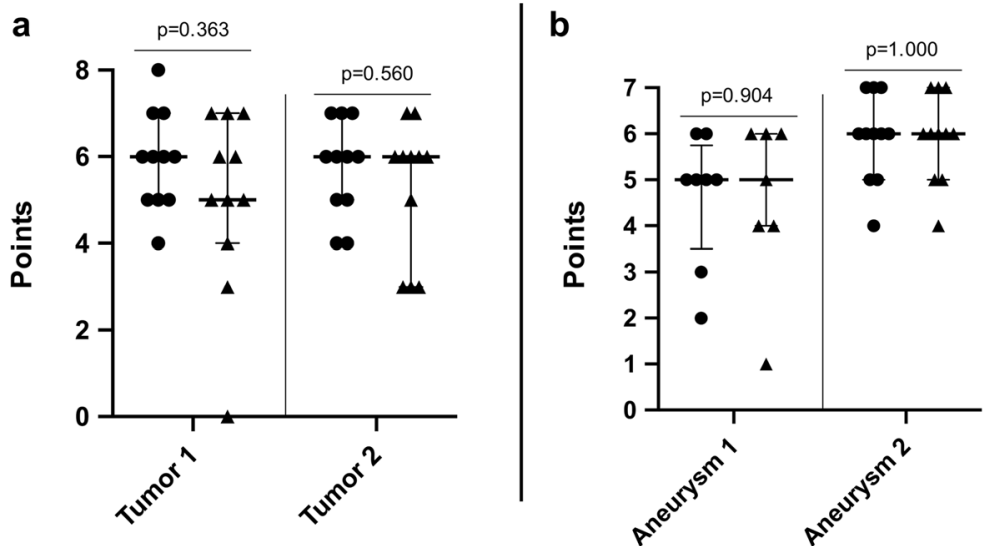

- Monoscopic

- Stereoscopic

Fig. 5 a Comparison of the achieved points in evaluating the tumor patients for monoscopic and stereoscopic imaging systems with 11 participants for each tumor (Mann-Whitney $U$ test). The dots and triangles represent the achieved points by each participant for both tumors; the lines represent the median and the interquartile range (25th-75th percentile). b Comparison of the achieved points in evalu- ating the aneurysm patients for monoscopic and stereoscopic imaging systems with 8 (monoscopic) and 7 (stereoscopic) participants for aneurysm 1 and 11 participants in each group for aneurysm 2 (MannWhitney $U$ test). The dots and triangles represent the achieved points by each participant for both aneurysms; the lines represent the median and the interquartile range (25th-75th percentile)

Table 2 Itemized objective analysis comparison of residents and consultants for both tumors (T1 and T2) and aneurysms (A1 and A2)

\begin{tabular}{llllllllll}
\hline & T1 mono & T1 stereo & T2 mono & T2 stereo & A1 mono & A1 stereo & A2 mono & A2 stereo \\
\hline Residents $(n=8)^{*}$ & Median & 6 & 5 & 5.5 & 6 & $5(n=6)$ & $5(n=5)$ & 6 & 6 \\
& 25th percentile & 5 & 3.75 & 4.75 & 4.5 & 3.5 & 4 & 5.75 & 5 \\
& 75th percentile & 7 & 6.25 & 6 & 6 & 5 & 6 & 6.25 & 6.25 \\
Consultants $(n=3)^{*}$ & Median & 6 & 6 & 7 & 6 & $5.5(n=2)$ & $5(n=2)$ & 6 & 6 \\
& 25th percentile & 5.5 & 5.5 & 6.5 & 4.5 & 5.25 & 4.5 & 5.5 & 6 \\
& 75th percentile & 6 & 6.5 & 7 & 6.5 & 5.75 & 5.5 & 6.5 & 6.5 \\
\hline
\end{tabular}

*Number of participants is stated, if it differs from here

imaging modalities, the time to detect the aneurysms was in the same range (Fig. 6b).

When comparing residents and consultants, the most obvious difference was observed for the aneurysm detection in posterior circulation (aneurysm 1) using the stereoscopic imaging system: The consultants required $210 \mathrm{~s}$ in median [IQR: 180-240] to find the aneurysm, while the residents required only $110 \mathrm{~s}$ in median [IQR: 105-200] (Table 3). For the overall time needed for the analysis of aneurysm 1 using the monoscopic imaging system, the residents required more time (Table 4) (median [IQR]: residents: 255 [237.5-277.5] s, consultants: 150 [115-157.5] s).

\section{Correlation analysis}

The total points of each participant for both cases per visualization system were added in order to correlate the objective performance in the second questionnaire with the level of experience. The total points that the participants had achieved after using the monoscopic or stereoscopic imaging system were not correlated to their experience level (Fig. 7).

\section{Discussion}

In this study, we analyzed the value of stereoscopic visualization for memorization on the basis of four different neurosurgical cases in a group of 22 neurosurgeons with various experience levels. The results demonstrate that the accuracy of stereoscopic assessment of neurosurgical pathologies was comparable to the monoscopic visualization used routinely. However, the subjective perception ranked the stereoscopic imaging worse, most likely due to the lack of prior experience with 3D imaging in general and the used system in particular.

The use of three-dimensional non-stereoscopic reconstructions has already been investigated in several studies in different fields [17-19]. For instance, students that learned 
Duration of the Pathology Assessment

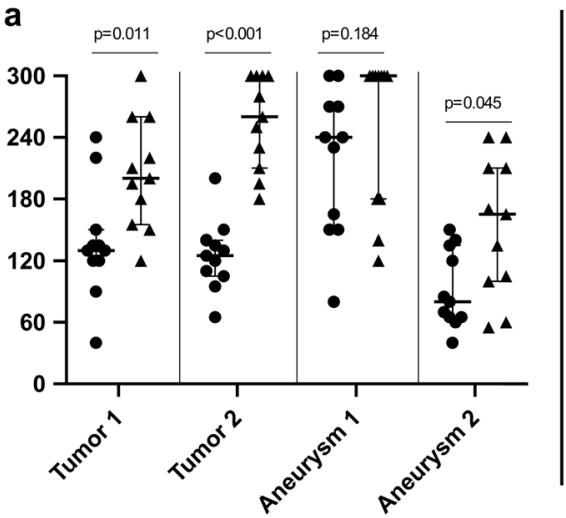

Fig. 6 a Comparison of the time to evaluate the different pathologies for monoscopic and stereoscopic imaging system with 11 participants for each pathology and imaging system. The time to analyze the pathologies was significantly less using the monoscopic imaging system except in aneurysm 1 (Mann-Whitney $U$ test). The dots and triangles represent the time needed by each participant to analyze the different pathologies; the lines represent the median and the inter-

b

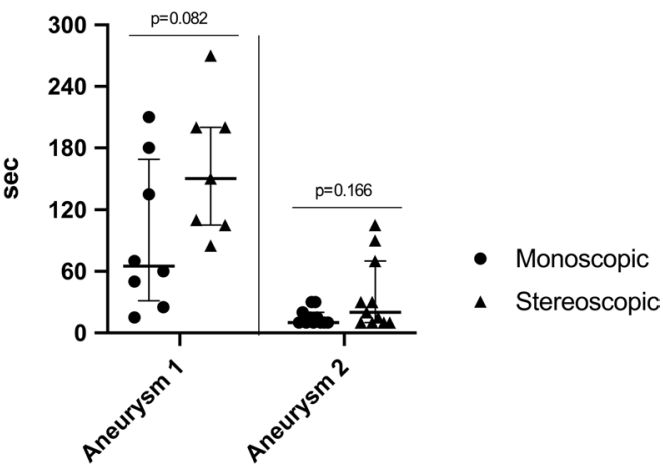

quartile range (25th-75th percentile). b Comparison of the time to detect the aneurysm for monoscopic and stereoscopic imaging systems with 8 (monoscopic) and 7 (stereoscopic) participants for aneurysm 1 and 11 participants for aneurysm 2 (Mann-Whitney $U$ test). The dots and triangles represent the time needed by each participant to find the aneurysm; the lines represent the median and the interquartile range (25th-75th percentile)
Table 3 Itemized time todetect the aneurysms (in seconds)with comparison of residents and consultants

\begin{tabular}{llllll}
\hline & A1 mono & A1 stereo & A2 mono & A2 stereo \\
\hline Residents & Median & $102.5(n=6)$ & $110(n=5)$ & $15(n=8)$ & $15(n=8)$ \\
& 25th percentile & 62.5 & 105 & 10 & 10 \\
\multirow{4}{*}{ Consultants } & 75th percentile & 168.75 & 200 & 22.5 & 30 \\
& Median & $37.5(n=2)$ & $210(n=2)$ & $10(n=3)$ & $70(n=3)$ \\
& 25th percentile & 31.25 & 180 & 10 & 42.5 \\
& 75th percentile & 43.75 & 240 & 10 & 87.5 \\
\hline
\end{tabular}

Table 4 Itemized time to analyze the pathologies (in seconds) with comparison of residents and consultants

\begin{tabular}{llllllllll}
\hline & T1 mono & T1 stereo & T2 mono & T2 stereo & A1 mono & A1 stereo & A2 mono & A2 stereo \\
\hline Residents $(n=8)$ & Median & 132.5 & 205 & 122.5 & 265 & 255 & 300 & 82.5 \\
& 25th percentile & 112.5 & 191.25 & 106.25 & 206.25 & 237.5 & 180 & 68.75 & 90 \\
& 75th percentile & 167.5 & 230 & 138.75 & 300 & 277.5 & 300 & 136.25 & 217.5 \\
Consultants $(n=3)$ & Median & 130 & 150 & 130 & 260 & 150 & 300 & 65 & 165 \\
& 25th percentile & 125 & 135 & 117.5 & 245 & 115 & 220 & 62.5 & 135 \\
& 75th percentile & 132.5 & 205 & 135 & 280 & 157.5 & 300 & 92.5 & 187.5 \\
\hline
\end{tabular}

gastrointestinal anatomy with 3D reconstructions achieved superior test results and were more skillful at dissection [19]. Glittenberg et al. demonstrated that 3D animations improved the understanding of ophthalmic anatomy and physiology [18]. For neurosurgery, Kikinis et al. reported the benefit of three-dimensional visualization to gain otherwise almost inaccessible information about the spatial relations that could be used for the surgical removal of brain lesions [16]. A systematic review by Arantes et al. concluded that 3D models were an effective way to teach neuroanatomy [20].
Despite these studies demonstrating the advantages of 3D visualization in teaching and preoperative planning, Park et al. could not detect a benefit of the three-dimensional anatomical atlas for the memorization of anatomical structures for first-year medical students [13]. Stereoscopy on the other hand offers a better depth sensation of the 3D images. Additionally, according to the cognitive load theory, more mental connections are activated during stereoscopic viewing that could possibly support the memorization process [21]. Stereoscopic imaging systems have also proven to demonstrate 


\section{Correlation}

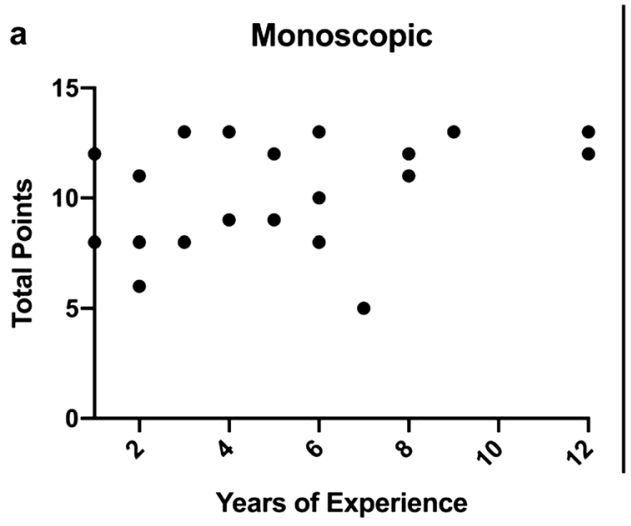

Fig. 7 a Correlation of the years of experience and the total points achieved using the monoscopic visualization system (Spearman rank correlation coefficient; $\rho=0.289 ; p=0.193$ ). The dots represent each participant's achieved points and their year of experience. b Correla-

a significant improvement in the acquisition of minimally invasive surgical skills for novice surgeons as compared to the standard 2D imaging system [22-24]. For instance, in a study by Schoenthaler et al. utilizing a surgical simulator model for laparoscopic surgery, stereoscopic imaging led to an improvement while performing the laparoscopic phantom tasks [22]. Likewise, advantages of stereoscopic threedimensional imaging in teaching anatomy have already been identified in the past [15]. Stadie et al. were able to show that through virtual reality planning the spatial understanding of the anatomy of neurosurgical cases could be improved in $55.8 \%$ of the participants and only $2.4 \%$ reported no improvement [25]. Brown et al. have also demonstrated that subjectively students achieved a better understanding of anatomy and pathology through stereoscopic three-dimensional images [26]. Furthermore, a recently published metaanalysis focusing on the anatomy learning supported these findings and highlighted the advantage of stereoscopic 3D over non-stereoscopic 3D [14]. However, the potential of three-dimensional volume rendering for stereoscopic visualization has not been fully exploited [27]. Despite these studies in support of stereoscopic visualization in education, Chytas et al. recently discussed the methodological inadequacy of some of the previously published comparative studies and encouraged further investigations [28].

An important aspect of evaluating the usefulness of stereoscopy is one's experience in the field and the area of application being examined. In both above-mentioned studies, the advantages of the stereoscopy in teaching were mainly seen amongst students who had no professional experience in clinical routine $[15,26]$. On one hand, since the younger generation is exposed to three-dimensional visualization much earlier, they can naturally feel more familiar

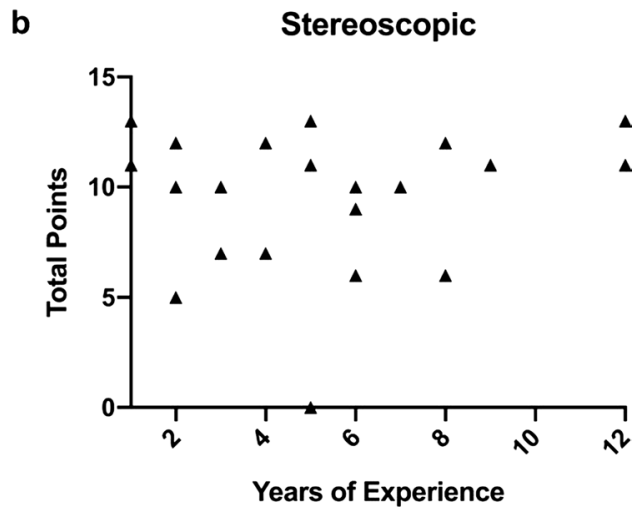

tion of the years of experience and the total points achieved using the stereoscopic visualization system (Spearman rank correlation coefficient; $\rho=-0.005 ; p=0.982$ ). The dots represent each participant's achieved points and their year of experience

and confident with this type of imaging. On the other hand, the advantage of stereoscopy in diagnostics, for example, could wane with increasing professional experience in the application of monoscopic visualizations, which enables a better understanding of 2D imaging and the ability to reconstruct images in the head. Consistent with this assumption, we could only find a benefit in detecting aneurysms using stereoscopic visualization for residents and not for consultants in our previous study [8]. However, due mainly to the limited number of participants, we were unable to detect any correlation between the years of experience and the points achieved in the present study. This was also supported by the lack of significant differences in individual categories between the residents and consultants. In this context, however, we would like to point out that stereoscopy has a wide range of applications in medicine that is not limited to diagnostics or teaching. During an operation in particular, the sense of depth is of great importance when using a surgical microscope, in which stereoscopy certainly retains its advantages regardless of experience level.

Importantly, both assessment groups (A and B) in our study were overall comparable regarding neurosurgical experience and stereoacuity. The capacity to perceive stereoscopic images is the primary requirement for stereoscopy. According to Odell et al., a stereoacuity of $>250 \mathrm{~s}$ arc is considered insufficient [29]. All of our participants had sufficient stereopsis, that highlights the possible routine application of this technique in daily clinical life. This can be supported by the results of other studies for surgeons in which only $2-14 \%$ (depending on the test) of the participating surgeons had impaired stereopsis [30]. Furthermore, Chopin et al. estimated the prevalence of "stereoblindness" in adults under 60 years is only about 7\% [31]. 
One major issue in the implementation of stereoscopic visualization in the clinical routine for image assessments, nevertheless, is the prerequisite training required to familiarize the user with this or similar specific systems. Importantly, neurosurgeons are usually familiar with deep immersion through stereoscopic visualization, as it is routinely used in the surgical microscope. Adaptation to stereoscopic viewing is therefore not a major problem. However, different handling is required for the evaluation of the MRI or CT imaging with stereoscopic visualization. In the subjective analysis of our participants, the stereoscopic visualization seemed to have no advantages over the conventional imaging technique. In fact, using the new system turned out to be more complicated and less intuitive for the majority, especially in handling. This could be partly explained by a lack of prior training with this specific system, particularly with regard to scrolling with the $3 \mathrm{D}$ mouse. It is also of interest that participants rated the stereoscopic presentation of aneurysm cases better than that of the tumor cases. A similar result was also shown by Bairamian et al., who observed an advantage of stereoscopy in the visualization of challenging cerebrovascular anatomy [32]. This might be due to the better visualization of the vessels by colored contrast adjustments. Overall, the data so far indicate a benefit of stereoscopic visualization in the diagnostic way for selected cases, so this new technology should be implemented as a supplement to conventional $2 \mathrm{D}$ visualization.

It is of major importance that the objective results did not coincide with the subjective assessment that the pathologies were worse to assess. In the objective analysis, the stereoscopic visualization was not inferior to the monoscopic one despite lack of prior training with the new system. The individual pathologies were assessed comparable by both groups using both systems. As the pathologies themselves were not easily comparable, an analysis between the two systems per participant as performed in our previous study was omitted here [8]. For instance, in contrast to the second aneurysm, the first aneurysm could not be detected by all participants. Contrary to our previous results, more participants during the stereoscopic visualization system session were unable to find the aneurysm in the posterior circulation [8]. This could be because the participants in this study had to scroll through the images themselves. The video in the Appendix illustrates an appropriate overview of the aneurysms (video 1).

The lack of familiarity with the stereoscopic system could also explain the longer time required to analyze the cases. A longer processing time was also revealed by the results of the examination of 3D echocardiography [7]. Even so, the aneurysms were diagnosed equally quickly on both imaging systems and the residents were able to identify both aneurysm cases faster using the stereoscopic system compared to the consultants. This may be due to the variation in exposure of different generational cohorts to such technologies. On the other hand, Itatani et al. stated that stereoscopic visualization could reduce the operative time in laparoscopic gastrectomy when used by trained surgeons [33]. In our study, all expertise levels handled the monoscopic imaging system more quickly, presumably due to its use in the routine clinical practice and a lack of training with the newer technology.

The main limitation of our study is the low number of participants and cases presented due to its monocentric design. In addition, the lack of prior training with this specific stereoscopic system may have diminished the potential benefits of the new imaging modality. In this context, we noticed that even in the stereoscopic session, participants tended to use the conventional levels during image assessments. This could also be one of the reasons that the full potential of stereoscopic imaging may not have been verified. Another potential point of criticism is the comparison between the stereoscopic 3D imaging with only 2D images as discussed by Chytas et al. [28]; however, our goal was to evaluate the added value of stereoscopy to the $2 \mathrm{D}$ images in clinical routine.

\section{Conclusion}

Monoscopic visualization was preferred by most study participants in comparison to the unaccustomed stereoscopic system. Stereoscopic imaging, however, was not inferior to conventional monoscopic imaging for memorization. In the future, more methodological developments and incorporation in routine clinical workflows will most likely increase the usability and acceptance of stereoscopic visualization. Therefore, further, preferably multicenter studies on this topic are warranted.

Supplementary Information The online version contains supplementary material available at https://doi.org/10.1007/s10143-021-01623-0.

Acknowledgements Dr. Acker is a participant of the BIH-Charité Clinician Scientist Program that is funded by the Charite Universitätsmedizin Berlin and the Berlin Institute of Health.The authors acknowledge the support of the Cluster of Excellence Matters of Activity. Image Space Material was funded by the Deutsche Forschungsgemeinschaft (DFG, German Research Foundation) under Germany's Excellence Strategy—EXC 2025 - 390648296.

Author contribution Nicolas Schlinkmann, Lucius S. Fekonja, Thomas Picht and Güliz Acker contributed to the study conception and design. Material preparation and data collection were done by Nicolas Schlinkmann, and analysis was performed by Nicolas Schlinkmann, Sophie K. Piper and Güliz Acker. The first draft of the manuscript was written by Nicolas Schlinkmann, revised critically for important intellectual content by Rutvik Khakhar, Thomas Picht, Lucius S. Fekonja, Peter Vajkoczy, and Güliz Acker. All authors commented on previous versions of the manuscript. All authors read and approved the final manuscript.

Availability of data and material The datasets generated during and/or analyzed during the current study are available from the corresponding author on reasonable request. 
Code availability Not applicable.

\section{Declarations}

Ethics approval Ethics committee of Charité - Universitätsmedizin Berlin (EA1/090/20)

Consent to participate Verbal informed consent was obtained prior to the interview.

Consent for publication Not applicable.

Conflict of interest The authors declare no competing interests.

Open Access This article is licensed under a Creative Commons Attribution 4.0 International License, which permits use, sharing, adaptation, distribution and reproduction in any medium or format, as long as you give appropriate credit to the original author(s) and the source, provide a link to the Creative Commons licence, and indicate if changes were made. The images or other third party material in this article are included in the article's Creative Commons licence, unless indicated otherwise in a credit line to the material. If material is not included in the article's Creative Commons licence and your intended use is not permitted by statutory regulation or exceeds the permitted use, you will need to obtain permission directly from the copyright holder. To view a copy of this licence, visit http://creativecommons.org/licenses/by/4.0/.

\section{References}

1. Handels H, Ehrhardt J, Plotz W, Poppl SJ (2001) Three-dimensional planning and simulation of hip operations and computerassisted construction of endoprostheses in bone tumor surgery. Comput Aided Surg 6(2):65-76. https://doi.org/10.1002/igs.1010

2. Jacquesson T, Simon E, Dauleac C, Margueron L, Robinson P, Mertens P (2020) Stereoscopic three-dimensional visualization: interest for neuroanatomy teaching in medical school. Surg Radiol Anat. https://doi.org/10.1007/s00276-020-02442-6

3. Liu X, Tao H, Xiao X, Guo B, Xu S, Sun N, Li M, Xie L, Wu C (2018) Use of the stereoscopic virtual reality display system for the detection and characterization of intracranial aneurysms: a Icomparison with conventional computed tomography workstation and 3D rotational angiography. Clin Neurol Neurosurg 170:93-98. https://doi.org/10.1016/j.clineuro.2018.04.034

4. Rubio RR, Bonaventura RD, Kournoutas I, Barakat D, Vigo V, El-Sayed I, Abla AA (2020) Stereoscopy in surgical neuroanatomy: past, present, and future. Oper Neurosurg (Hagerstown) 18(2):105-117. https://doi.org/10.1093/ons/opz123

5. Storz P, Buess GF, Kunert W, Kirschniak A (2012) 3D HD versus 2D HD: surgical task efficiency in standardised phantom tasks. Surg Endosc 26(5):1454-1460. https://doi.org/10.1007/ s00464-011-2055-9

6. Meyer ER, Cui D (2020) Anatomy visualizations using stereopsis: assessment and implication of stereoscopic virtual models in anatomical education. Adv Exp Med Biol 1235:117-130. https:// doi.org/10.1007/978-3-030-37639-0_7

7. Harake D, Gnanappa GK, Alvarez SGV, Whittle A, Punithakumar K, Boechler P, Noga M, Khoo NS (2020) Stereoscopic display is superior to conventional display for three-dimensional echocardiography of congenital heart anatomy. J Am Soc Echocardiogr 33(11):1297-1305. https://doi.org/10.1016/j.echo.2020.06.016
8. Acker G, Schlinkmann N, Piper SK, Onken J, Vajkoczy P, Picht $\mathrm{T}$ (2018) Stereoscopic versus monoscopic viewing of aneurysms: experience of a single institution with a novel stereoscopic viewing system. World Neurosurg 119:e491-e501. https://doi.org/10. 1016/j.wneu.2018.07.189

9. Stewart N, Lock G, Hopcraft A, Kanesarajah J, Coucher J (2014) Stereoscopy in diagnostic radiology and procedure planning: does stereoscopic assessment of volume-rendered CT angiograms lead to more accurate characterisation of cerebral aneurysms compared with traditional monoscopic viewing? J Med Imaging Radiat Oncol 58(2):172-182. https://doi.org/10.1111/1754-9485.12146

10. Alsaid B, Bertrand M (2016) Students' memorization of anatomy, influence of drawing. Morphologie 100(328):2-6. https://doi.org/ 10.1016/j.morpho.2015.11.001

11. Langridge B, Momin S, Coumbe B, Woin E, Griffin M, Butler P (2018) Systematic review of the use of 3-dimensional printing in surgical teaching and assessment. J Surg Educ 75(1):209-221. https://doi.org/10.1016/j.jsurg.2017.06.033

12. Spiriev T, Nakov V, Laleva L, Tzekov C (2017) OsiriX software as a preoperative planning tool in cranial neurosurgery: a stepby-step guide for neurosurgical residents. Surg Neurol Int 8:241. https://doi.org/10.4103/sni.sni_419_16

13. Park S, Kim Y, Park S, Shin JA (2019) The impacts of threedimensional anatomical atlas on learning anatomy. Anat Cell Biol 52(1):76-81. https://doi.org/10.5115/acb.2019.52.1.76

14. Bogomolova K, Hierck BP, Looijen AEM, Pilon JNM, Putter H, Wainman B, Hovius SER, van der Hage JA (2020) Stereoscopic three-dimensional visualisation technology in anatomy learning: a meta-analysis. Med Educ. doi:https://doi.org/10.1111/medu. 14352

15. Bernard F, Richard P, Kahn A, Fournier HD (2020) Does 3D stereoscopy support anatomical education? Surg Radiol Anat 42(7):843-852. https://doi.org/10.1007/s00276-020-02465-z

16. Kikinis R, Gleason PL, Moriarty TM, Moore MR, Alexander E, 3rd, Stieg PE, Matsumae M, Lorensen WE, Cline HE, Black PM, Jolesz FA (1996) Computer-assisted interactive three-dimensional planning for neurosurgical procedures. Neurosurgery 38(4):640 649; discussion 649-651

17. Sardari Nia P, Olsthoorn JR, Heuts S, Maessen JG (2019) Interactive $3 \mathrm{D}$ reconstruction of pulmonary anatomy for preoperative planning, virtual simulation, and intraoperative guiding in video-assisted thoracoscopic lung surgery. Innovations (Phila) 14(1):17-26. https://doi.org/10.1177/1556984519826321

18. Glittenberg C, Binder S (2006) Using 3D computer simulations to enhance ophthalmic training. Ophthalmic Physiol Opt 26(1):40 49. https://doi.org/10.1111/j.1475-1313.2005.00358.x

19. Tam MD, Hart AR, Williams SM, Holland R, Heylings D, Leinster S (2010) Evaluation of a computer program ('disect') to consolidate anatomy knowledge: a randomised-controlled trial. Med Teach 32(3):e138-142. https://doi.org/10.3109/014215909031441 10

20. Arantes M, Arantes J, Ferreira MA (2018) Tools and resources for neuroanatomy education: a systematic review. BMC Med Educ 18(1):94. https://doi.org/10.1186/s12909-018-1210-6

21. Sweller J (1988) Cognitive load during problem-solving - effects on learning. Cogn Sci 12(2):257-285. https://doi.org/10.1016/ 0364-0213(88)90023-7

22. Schoenthaler M, Schnell D, Wilhelm K, Schlager D, Adams F, Hein S, Wetterauer U, Miernik A (2016) Stereoscopic (3D) versus monoscopic (2D) laparoscopy: comparative study of performance using advanced HD optical systems in a surgical simulator model. World J Urol 34(4):471-477. https://doi.org/10.1007/ s00345-015-1660-y

23. Smith R, Day A, Rockall T, Ballard K, Bailey M, Jourdan I (2012) Advanced stereoscopic projection technology significantly improves novice performance of minimally invasive surgical 
skills. Surg Endosc 26(6):1522-1527. https://doi.org/10.1007/ s00464-011-2080-8

24. Wehr F, Held J (2019) Stereoscopic versus monoscopic displays: Learning fine manual dexterity skills using a microsurgical task simulator. Appl Ergon 77:40-49. https://doi.org/10.1016/j.apergo. 2019.01.006

25. Stadie AT, Kockro RA (2013) Mono-stereo-autostereo: the evolution of 3-dimensional neurosurgical planning. Neurosurgery 72(Suppl 1):63-77. https://doi.org/10.1227/NEU.0b013e3182 $70 \mathrm{~d} 310$

26. Brown PM, Hamilton NM, Denison AR (2012) A novel 3D stereoscopic anatomy tutorial. Clin Teach 9(1):50-53. https://doi. org/10.1111/j.1743-498X.2011.00488.x

27. Jha DK, Khera P, Bhaskar S, Garg M (2019) Three-dimensional volume rendering: an underutilized tool in neurosurgery. World Neurosurg 130:485-492. https://doi.org/10.1016/j.wneu.2019.07. 065

28. Chytas D, Piagkou M, Natsis K (2020) Does 3D stereoscopy support anatomical education? Surg Radiol Anat. https://doi.org/10. 1007/s00276-020-02588-3

29. Odell NV, Hatt SR, Leske DA, Adams WE, Holmes JM (2009) The effect of induced monocular blur on measures of stereoacuity.
J AAPOS 13(2):136-141. https://doi.org/10.1016/j.jaapos.2008. 09.005

30. Biddle M, Hamid S, Ali N (2014) An evaluation of stereoacuity (3D vision) in practising surgeons across a range of surgical specialities. Surgeon 12(1):7-10. https://doi.org/10.1016/j.surge. 2013.05.002

31. Chopin A, Bavelier D, Levi DM (2019) The prevalence and diagnosis of 'stereoblindness' in adults less than 60 years of age: a best evidence synthesis. Ophthalmic Physiol Opt 39(2):66-85. https:// doi.org/10.1111/opo.12607

32. Bairamian D, Liu S, Eftekhar B (2019) Virtual reality angiogram vs 3-dimensional printed angiogram as an educational tool-a comparative study. Neurosurgery 85(2):E343-E349. https://doi.org/10. 1093/neuros/nyz003

33. Itatani Y, Obama K, Nishigori T, Ganeko R, Tsunoda S, Hosogi H, Hisamori S, Hashimoto K, Sakai Y (2019) Three-dimensional stereoscopic visualization shortens operative time in laparoscopic gastrectomy for gastric cancer. Sci Rep 9(1):4108. https://doi.org/ 10.1038/s41598-019-40269-3

Publisher's note Springer Nature remains neutral with regard to jurisdictional claims in published maps and institutional affiliations. 